\title{
Classification of daily-life postural transitions using trunk- worn wearable barometric pressure sensor
}

\author{
Fabien Massé, Alan Kevin Bourke, Anisoara Paraschiv-lonescu, and Kamiar Aminian \\ Laboratory of Movement Analysis and Measurement (LMAM) \\ Ecole Polytechnique Fédérale de Lausanne (EPFL) \\ \{Fabien.Masse,Alan.Bourke,Anisoara.Ionescu,Kamiar.Aminian\}@epfl.ch
}

\begin{abstract}
Distinguishing sedentary from dynamic behavior is essential in addressing disease conditions that are influenced by mobility. Event-based activity recognition algorithms essentially rely on accurate classification of siting and standing postural transitions to distinguish whether the subject is sitting or standing. In this paper, the use of barometric pressure, to estimate altitude, is investigated. It enabled a correct classification of postural transitions with a sensitivity of $92.31 \%$ and specificity of $98.06 \%$. The type (sit-to-stand or stand-to-sit) of transition was also accurately identified.
\end{abstract}

\section{Keywords}

Activity, barometric pressure, wearable sensor, inertial sensor

\section{INTRODUCTION}

Many diseases, e.g. pain, obesity, neurological and orthopedic disorders affect the mobility of the subject. Distinguishing sedentary from dynamic behavior is essential in addressing such diseases. Event-based activity recognition algorithm [1] essentially relies on accurate classification of postural transition to distinguish whether the subject is sitting or standing. This has traditionally been done with inertial sensors but suffer from inaccuracy, especially in real word conditions. This paper aims to investigate a new method for sit-to-stand ( $\mathrm{SiSt}$ ) and stand-to-sit ( $\mathrm{StSi}$ ) postural transition detection and classification using a single inertial/barometric pressure sensor attached to the trunk.

\section{METHODS}

\subsection{Data collection and hardware}

The measuring system consisted of a wearable sensor on the trunk (a 6D inertial sensor, LSM330D, ST Micro, CH and a barometric pressure sensor, MS5611-BA01,Meas Specialties, CH) specially selected for its very-low noise. For validation purpose, two more inertial sensors were placed on thigh and shank, which with a validated algorithm provide actual postural transition [2]. Data were acquired at $200 \mathrm{~Hz}$ for the inertial and $25 \mathrm{~Hz}$ for pressure sensors. Four healthy participants $(27.5 \pm 3.5$ years / height: 180.2 $\pm 12.2 \mathrm{~cm}$ ) continuously wore the system for at least $6 \mathrm{~h}$ and performed in real-world conditions their daily activity.

\subsection{Data processing}

First, using the trunk sensor, the postural transition candidate is detected when the wavelet-filtered trunk angle exceeds a certain threshold [1]. A logistic regression (LR) is defined based on the range of the Savitzky-Golay filtered pressure and the absolute maximum of CWT-filtered pressure signal $(\mathrm{Haar} / \mathrm{scale}=150)$ around the transitions. LR is used to separate true (actual) from false transitions as it provides the probability $\left(\mathrm{P}_{\mathrm{Tr}}\right)$ of a candidate transition to be a true transition. Transition candidates having a $\mathrm{P}_{T r}>0.5$ are considered as true transitions. The type of transition ( $\mathrm{SiSt} / \mathrm{StSi}$ ) was classified on the sign of difference in pressure before and after each transition. For validation, actual transitions were obtained from the algorithm in [2]. Sensitivity (SEN), and specificity (SPE) were computed using a 10-fold cross-validation.

\section{RESULTS}

A total of $35 \mathrm{~h} / 91$ transitions were obtained. The results of the classification algorithm, which distinguishes true from false transitions, are listed in Table 1. The type of transitions was always classified correctly yielding an accuracy of $100 \%$.

Table 1. Postural transition classification

\begin{tabular}{|l|c|c|c|}
\hline \multicolumn{2}{|c|}{ Actual postural transition } & Positive & Negative \\
\hline $\begin{array}{l}\text { Detected } \\
\text { postural } \\
\text { transition }\end{array}$ & Positive & 84 & 5 \\
\cline { 2 - 4 } & Negative & 7 & 356 \\
\hline \multicolumn{2}{|l}{} & $\mathrm{SEN}=92.31 \%$ & $\mathrm{SPE}=98.06 \%$ \\
\hline
\end{tabular}

\section{DISCUSSION AND CONCLUSION}

Postural transitions were detected using inertial sensors and then classified using barometric pressure information. Most of the errors in selection stems from either delays in detection or a smaller height difference during transition, e.g., when sitting on a table top rather than a chair. An additional activity layer which takes as an input the transitions may improve activity recognition.

The excellent results suggest that barometric pressure is suitable for supplementing postural transition classification in daily-life.

\section{ACKNOWLEDGMENTS}

This study was partially supported by Rehabilitatitve Wayout In Responsive home Environments (REWIRE). REWIRE funded by the Seventh Framework Program (FP7), contract N. 287713.

\section{REFERENCES}

[1] A. Salarian, et al., "Ambulatory Monitoring of Physical Activities in Patients With Parkinson's Disease," Biomedical Engineering, IEEE Transactions on, vol. 54, no. 12, 2007.

[2] A. Paraschiv-Ionescu, et al., "Nonlinear analysis of human physical activity patterns in health and disease," Phys Rev E Stat Nonlin Soft Matter Phys, vol. 77, no. 2 Pt 1, Feb, 2008. 